\title{
Use of genotype $\times$ environment interaction model to accommodate genetic heterogeneity for residual feed intake, dry matter intake, net energy in milk, and metabolic body weight in dairy cattle
}

\author{
C. Yao, ${ }^{* 1}$ G. de los Campos, $†$ M. J. VandeHaar, $†$ D. M. Spurlock, $\ddagger$ L. E. Armentano, ${ }^{*}$ M. Coffey,§ Y. de Haas,\# \\ R. F. Veerkamp,\# C. R. Staples, II E. E. Connor,ף Z. Wang, ${ }^{* *}$ M. D. Hanigan,†† R. J. Tempelman, $†$ \\ and K. A. Weigel* \\ *Department of Dairy Science, University of Wisconsin, Madison 53706 \\ †Department of Animal Science, Michigan State University, East Lansing 48824 \\ ‡Department of Animal Science, lowa State University, Ames 50011 \\ $\S$ Scottish Agricultural College, Easter Bush, Midlothian, EH25 9RG, United Kingdom \\ \#Wageningen UR Livestock Research, Wageningen, $6700 \mathrm{AH}$, the Netherlands \\ IIDepartment of Animal Sciences, University of Florida, Gainesville 32611 \\ TAnimal Genomics and Improvement Laboratory, Agricultural Research Service, USDA, Beltsville, MD 20705 \\ ${ }^{* *}$ Department of Agricultural, Food and Nutritional Science, University of Alberta, Edmonton, AB, T6G 2P5, Canada \\ ††Department of Dairy Science, Virginia Tech, Blacksburg 24061
}

\section{ABSTRACT}

Feed efficiency in dairy cattle has gained much attention recently. Due to the cost-prohibitive measurement of individual feed intakes, combining data from multiple countries is often necessary to ensure an adequate reference population. It may then be essential to model genetic heterogeneity when making inferences about feed efficiency or selecting efficient cattle using genomic information. In this study, we constructed a marker $\times$ environment interaction model that decomposed marker effects into main effects and interaction components that were specific to each environment. We compared environment-specific variance component estimates and prediction accuracies from the interaction model analyses, an across-environment analyses ignoring population stratification, and a within-environment analyses using an international feed efficiency data set. Phenotypes included residual feed intake, dry matter intake, net energy in milk, and metabolic body weight from 3,656 cows measured in 3 broadly defined environments: North America (NAM), the Netherlands (NLD), and Scotland (SAC). Genotypic data included 57,574 single nucleotide polymorphisms per animal. The interaction model gave the highest prediction accuracy for metabolic body weight, which had the largest estimated heritabilities ranging from 0.37 to 0.55 . The withinenvironment model performed the best when predicting residual feed intake, which had the lowest estimated

Received June 13, 2016.

Accepted November 22, 2016 .

${ }^{1}$ Corresponding author: Chen.Yao225@gmail.com heritabilities ranging from 0.13 to 0.41 . For traits (dry matter intake and net energy in milk) with intermediate estimated heritabilities ( 0.21 to 0.50 and 0.17 to 0.53 , respectively), performance of the 3 models was comparable. Genomic correlations between environments also were computed using variance component estimates from the interaction model. Averaged across all traits, genomic correlations were highest between NAM and NLD, and lowest between NAM and SAC. In conclusion, the interaction model provided a novel way to evaluate traits measured in multiple environments in which genetic heterogeneity may exist. This model allowed estimation of environment-specific parameters and provided genomic predictions that approached or exceeded the accuracy of competing within- or acrossenvironment models.

Key words: genomic selection, interaction model, feed efficiency

\section{INTRODUCTION}

Feed efficiency in dairy cattle has gained the attention of researchers, dairy farmers, and breeding companies internationally. Early studies aimed to estimate variance components for residual feed intake (RFI; Van Arendonk et al., 1991; Veerkamp et al., 1995), identify additive and epistatic SNP associated with RFI (Yao et al., 2013), and combine health history data with SNP genotypes to predict future phenotypes (Yao et al., 2015). These studies were based on a few hundred animals from a single research station due to the exorbitant cost associated with measuring individual feed intakes. Next, scientists combined data collected under similar 
climate and management conditions to estimate genetic parameters for RFI (Williams et al., 2011; Hardie et al., 2015) and to predict estimated breeding values for RFI (Pryce et al., 2012; Davis et al., 2014). To further improve the reliability of prediction, researchers pooled data globally. Berry et al. (2014) estimated genetic parameters for DMI, and Tempelman et al. (2015) estimated overall and country-specific heritabilities of RFI and explored heterogeneous relationships between RFI and traits relating to energy utilization. Both studies concluded that genetic evaluation using data collated from international populations appeared promising. The challenge lies in considering the heterogeneous genetic variance components and potential genotype $x$ environment interactions when combining data from multiple sources.

Multiple statistical models have been explored to address the genetic heterogeneity of data consisting of several subpopulations (e.g., multienvironment or multibreed data). One simple option is to analyze subsets of data within each subpopulation separately (i.e., within-environment or within-breed analyses). A major drawback is that this method reduces sample size, and therefore hampers the accuracy of genomic selection. Additionally, this approach does not allow sharing of information between subpopulations, which is important if marker effects are correlated across subpopulations. A second option is to combine data across subpopulations and ignore potential heterogeneity (i.e., across-environment or across-breed analyses). This method increases sample size and ensures sharing of information across subpopulations, but assumes that marker effects are constant across subpopulations, which may limit the potential benefits of a combined analyses. Hayes et al. (2009) compared the prediction accuracy of a multibreed analyses with that of a withinbreed analyses using data from Holsteins and Jerseys. Those authors found no benefits of a combined analysis on prediction accuracy for the breed with a larger reference panel (Holstein), and observed that gains were minimal in prediction accuracy for the group with a smaller reference panel (Jersey).

Recently, de los Campos et al. (2015) suggested an intermediate multivariate marker $\times$ environment approach between the within-environment and acrossenvironment models (i.e., the interaction model) to tackle potential genetic heterogeneity. This approach explicitly model interactions between whole-genome markers and environments, which allows marker effects to be constant and group-specific across environments. It also provides estimates of genomic correlations between subpopulations for each trait, which can be used to assess genetic similarity between subpopulations.
The interaction model is also more straightforward to interpret and easier to implement than the traditional multivariate models (Karoui et al., 2012; Olson et al., 2012). Many studies in plant breeding have demonstrated the superiority of the interaction model relative to within-environment or across-environment analyses in terms of minimizing residual variance (Crossa et al., 2015) and increasing prediction accuracies of genomic selection (de los Campos et al., 2015; Lopez-Cruz et al., 2015); however, the interaction model failed to improve prediction accuracy in another recent study (Lehermeier et al., 2015). In the current study, the aforementioned interaction model was assessed and compared with within-environment and across-environment models using data from multiple environments to estimate genomic variances and assess the accuracy of genomic predictions for RFI and its component traits.

\section{MATERIALS AND METHODS}

\section{Phenotypic Data}

The data used in this study consisted of 3,656 cows from 3 broadly defined environments: 2,329 cows from North America (NAM), representing the United States and Canada; 875 cows from the Netherlands (NLD); and 452 cows from Scotland (SAC). More detailed information about the data sources can be found in Tempelman et al. (2015).

Phenotypic data included 4 traits, RFI, DMI, net energy in milk (MilkE), and metabolic body weight (MBW), per cow per day during the period from 50 to $200 \mathrm{~d}$ postpartum. Individual feed intakes were recorded daily using electronic measurement systems or manual weigh-backs. Dry matter percentage dried at $60^{\circ} \mathrm{C}$ was analyzed periodically (typically once a week), and individual DMI of cows were calculated weekly using the DM percentage of feed offered. According to NRC (2001), daily MilkE was calculated based on the gross energy per kilogram in fat, protein, and lactose as

$$
\begin{gathered}
\text { MilkE }(\text { Mcal })=(0.0929 \times \text { fat } \%+0.0563 \times \text { protein } \% \\
+0.0395 \times \text { lactose } \%) \times \text { daily milk yield }(\mathrm{kg}) . \quad[1]
\end{gathered}
$$

Body weight was typically measured weekly, and MBW was computed as BW to the 0.75 power.

Each trait was adjusted for known fixed and random factors using a linear mixed-effects model. Residual feed intake was calculated as the deviation of the feed intake from the expected feed intake as

$$
\mathbf{y}=\mathbf{X} \boldsymbol{\beta}+\mathbf{Z u}+\mathbf{e},
$$


where $\mathbf{y}$ was a vector of phenotype records of DMI; $\boldsymbol{\beta}$ was a vector of fixed effects, including herd-year-season at calving, parity-by-age at calving, DIM, MilkE, and MBW; $\mathbf{u} \sim N\left(0, \mathbf{I} \sigma_{u}^{2}\right)$ was a vector of random effects for the specific experiment that animals received treatments; $\mathbf{e} \sim N\left(0, \mathbf{I} \sigma_{e}^{2}\right)$ was a vector of random residuals, where $\sigma_{u}^{2}$ and $\sigma_{e}^{2}$ were corresponding variances for $u$ and $e$ and $\mathbf{I}$ was the identity matrix; and $\mathbf{X}$ and $\mathbf{Z}$ were design matrices relating the observations $\mathbf{y}$ to $\boldsymbol{\beta}$ and $\mathbf{u}$, respectively. For DMI, MilkE, and MBW, y was a vector of phenotypes for the given trait, respectively; $\boldsymbol{\beta}$ was a vector of fixed effects, including herd-year-season at calving, parity-by-age at calving, and DIM; $\mathbf{u}, \mathbf{e}, \mathbf{X}$ and $\mathbf{Z}$ were the same as above. Levels of parity-by-age were first parity $\leq 22,24,25,26$, or $\geq 27$ mo; second parity $\leq 35,36, \ldots, 39$, or $\geq 40$ mo; third parity and later $\leq 48,49,50,51,52$ to 59,60 to 69 , or $\geq 70 \mathrm{mo}$. The range of DIM was from 50 to $200 \mathrm{~d}$ after calving. The mixed model equations were solved with the restricted maximum likelihood (REML) method using the Rpackage "lme4" (Version 0.999999-0; Bates et al., 2013). Finally, the residual term e was equivalent to the daily RFI, DMI, MilkE, and MBW estimates, which were averaged for each cow to be the phenotype value used in the following genomic evaluation.

\section{Genotypic Data}

Single nucleotide polymorphism genotypes were available for all 3,656 cows. Genotypes were obtained from the Council on Dairy Cattle Breeding (Bowie, MD) Holstein genotype database. Raw genotypes represented various low- and medium-density arrays, and missing genotypes were imputed to higher density using genotype information from bulls and cows in the Council on Dairy Cattle Breeding database (Wiggans et al., 2014). Any remaining missing genotypes (about $2 \%$ ) were filled in using rounded allele frequencies from the current US Holstein population. The SNP genotype at each locus was coded as 0,1 , or 2 by counting the number of copies of the minor allele; SNP with minor allele frequencies less than $5 \%$ were removed. A total of 57,574 SNP per cow were available for the genomic evaluation analyses.

\section{Statistical Model}

We considered 3 whole-genome marker regression approaches for genomic evaluation: an interaction model that analyzed the data from multiple environments jointly and allowed marker by environments interactions; a within-environment analyses that regressed phenotypes on markers separately within each environment; and an across-environment analyses that assumed marker effects were constant across environments. Each approach is described below.

Interaction Model. The interaction model assumed that marker effects had 2 components, one that was common across all environments $\left(\beta_{0}\right)$ and one that was environment-specific $\left(\beta_{1}\right.$ and $\left.\beta_{2}\right)$. The interaction model could include subpopulations from all available environments or only a pair of environments. However, it imposed restrictions on covariance patterns: the covariance between environments was forced to be positive and constant across pairs of environments. Thus, the interaction model was best suited for the joint analyses of sets of environments that were positively and similarly correlated. In this study, we included 2 environments each time due to unknown correlations between environments. The equation was

$$
\left[\begin{array}{l}
y_{1} \\
y_{2}
\end{array}\right]=\left[\begin{array}{l}
1 \mu_{1} \\
1 \mu_{2}
\end{array}\right]+\left[\begin{array}{c}
X_{1} \\
X_{2}
\end{array}\right] \boldsymbol{\beta}_{0}+\left[\begin{array}{c}
X_{1} \\
0
\end{array}\right] \boldsymbol{\beta}_{1}+\left[\begin{array}{c}
0 \\
X_{2}
\end{array}\right] \boldsymbol{\beta}_{2}+\left[\begin{array}{c}
\varepsilon_{1} \\
\varepsilon_{2}
\end{array}\right],
$$

where $y_{1}, y_{2}, X_{1}$, and $X_{2}$ represented the phenotypes and genotypes of individuals in environment 1 and 2, respectively; $\mu_{1}$ and $\mu_{2}$ were environment-specific inter$\begin{array}{lll}\text { cepts; } & \boldsymbol{\beta}_{0} \sim N\left(0, \mathbf{G}_{0} \sigma_{g_{0}}^{2}\right), \quad \boldsymbol{\beta}_{1} \sim N\left(0, \mathbf{G}_{1} \sigma_{g_{1}}^{2}\right), \quad \text { and }\end{array}$ $\boldsymbol{\beta}_{2} \sim N\left(0, \mathbf{G}_{2} \sigma_{g_{2}}^{2}\right)$ were vectors of marker effects; $\varepsilon_{1} \sim N\left(0, \mathbf{I} \sigma_{\varepsilon_{1}}^{2}\right)$ and $\varepsilon_{2} \sim N\left(0, \mathbf{I} \sigma_{\varepsilon_{2}}^{2}\right)$ were model errors; $\sigma_{g_{0}}^{2}$ was common genomic variance, $\sigma_{g_{1}}^{2}$ and $\sigma_{g_{2}}^{2}$ were environment-specific genomic variances, and $\sigma_{\varepsilon_{1}}^{2}$ and $\sigma_{\varepsilon_{2}}^{2}$ were environment-specific residual variances. Genomic relationship matrices were computed as

$$
\begin{gathered}
\mathbf{G}_{0}=p^{-1}\left[\begin{array}{cc}
\mathbf{W}_{1} \mathbf{W}_{1}^{\prime} & \mathbf{W}_{1} \mathbf{W}_{2}^{\prime} \\
\mathbf{W}_{2} \mathbf{W}_{1}^{\prime} & \mathbf{W}_{2} \mathbf{W}_{2}^{\prime}
\end{array}\right], \\
\mathbf{G}_{1}=p^{-1} \mathbf{W}_{1} \mathbf{W}_{1}^{\prime}, \\
\mathbf{G}_{2}=p^{-1} \mathbf{W}_{2} \mathbf{W}_{2}^{\prime},
\end{gathered}
$$

and

where $p=57,574$ and $\mathbf{W}_{\mathbf{1}}$ and $\mathbf{W}_{\mathbf{2}}$ were matrices containing scaled and centered genotypes from $\mathbf{X}_{1}$ and $\mathbf{X}_{2}$; centering and scaling were performed based on the sample mean and sample SD computed using $\mathbf{X}_{1}$ and $\mathbf{X}_{2}$.

Within-Environment Model. The within-environment model performed genomic evaluation within each environment separately and could be obtained by assuming $\boldsymbol{\beta}_{0}=0$ from the interaction model, as follows: 
Table 1. Numbers of cows partitioned to the training and testing sets for validation assessment of prediction accuracy; environments included North America (NAM), the Netherlands (NLD), and Scotland (SAC)

\begin{tabular}{llrrr}
\hline Model & Environment & Training & Testing & Total \\
\hline Within-environment model & NAM & 1,863 & 466 & 2,329 \\
& NLD & 700 & 175 & 875 \\
\multirow{2}{*}{ Across-environment and interaction models } & SAC & 362 & 90 & 452 \\
& NLD-NAM & 2,563 & 641 & 3,204 \\
& SAC-NAM & 2,225 & 556 & 2,781 \\
& NLD-SAC & 1,062 & 265 & 1,327 \\
\hline
\end{tabular}

$$
\left[\begin{array}{l}
y_{1} \\
y_{2}
\end{array}\right]=\left[\begin{array}{c}
1 \mu_{1} \\
1 \mu_{2}
\end{array}\right]+\left[\begin{array}{c}
X_{1} \\
0
\end{array}\right] \boldsymbol{\beta}_{\mathbf{1}}+\left[\begin{array}{c}
0 \\
X_{2}
\end{array}\right] \boldsymbol{\beta}_{\mathbf{2}}+\left[\begin{array}{c}
\varepsilon_{1} \\
\varepsilon_{2}
\end{array}\right]
$$

Across-Environment Model. The across-environment model assumed that marker effects were common for all environments and could be obtained by setting $\boldsymbol{\beta}_{1}=\boldsymbol{\beta}_{2}=0$ from the interaction model. The equations became:

$$
\left[\begin{array}{l}
y_{1} \\
y_{2}
\end{array}\right]=\left[\begin{array}{l}
1 \mu_{1} \\
1 \mu_{2}
\end{array}\right]+\left[\begin{array}{l}
X_{1} \\
X_{2}
\end{array}\right] \boldsymbol{\beta}_{\mathbf{0}}+\left[\begin{array}{l}
\varepsilon_{1} \\
\varepsilon_{2}
\end{array}\right] .
$$

\section{Inference on Variance Component}

The complete data set was fitted to estimate the genomic variance components. The posterior means of common genomic variance $\left(\sigma_{g_{0}}^{2}\right)$, environment-specific genomic variances $\left(\sigma_{g_{1}}^{2}\right.$ and $\left.\sigma_{g_{2}}^{2}\right)$, and environment-specific residual variances $\left(\sigma_{\varepsilon_{1}}^{2}\right.$ and $\left.\sigma_{\varepsilon_{2}}^{2}\right)$ were reported for each trait. The genomic heritabilities of each trait, $h_{\text {int }_{i}}^{2}$, $h_{\mathrm{win}_{i}}^{2}$, and $h_{\mathrm{acr}_{i}}^{2}$ in environment $i(i=1$ and 2$)$, were calculated for the interaction model (int), within-environment model (win), and across-environment model (acr) as follows, respectively:

$$
\begin{aligned}
& h_{\mathrm{int}_{i}}^{2}=\left(\sigma_{g_{i}}^{2}+\sigma_{g_{0}}^{2}\right) /\left(\sigma_{g_{0}}^{2}+\sigma_{g_{i}}^{2}+\sigma_{\varepsilon_{i}}^{2}\right), \\
& h_{\text {win }_{i}}^{2}=\sigma_{g_{i}}^{2} /\left(\sigma_{g_{i}}^{2}+\sigma_{\varepsilon_{i}}^{2}\right), \text { and } \\
& h_{\text {acr }_{i}}^{2}=\sigma_{g_{0}}^{2} /\left(\sigma_{g_{0}}^{2}+\sigma_{\varepsilon_{i}}^{2}\right) .
\end{aligned}
$$

The trait-specific genomic correlations $\left(\operatorname{cor}_{g_{i, j}}\right)$ between $y_{1}$ and $y_{2}$ were also calculated from the interaction model as follows:

$\operatorname{cor}_{g_{1,2}}=\frac{\operatorname{cov}_{g}\left(y_{1}, y_{2}\right)}{\left[\operatorname{var}_{g}\left(y_{1}\right) \operatorname{var}_{g}\left(y_{2}\right)\right]^{1 / 2}}=\frac{\sigma_{g_{0}}^{2}}{\left[\left(\sigma_{g_{0}}^{2}+\sigma_{g_{2}}^{2}\right)\left(\sigma_{g_{0}}^{2}+\sigma_{g_{1}}^{2}\right)\right]^{1 / 2}}$.

\section{Assessment of Prediction Accuracy}

Prediction accuracy was assessed using the repeated holdout method. We randomly sampled $80 \%$ of data as the training set and tested prediction accuracy on the remaining $20 \%$ of data. The partitioning was repeated 10 times for each model to have more reliable prediction accuracies. The actual numbers of animals per environment in the training and testing sets by model are shown in Table 1. Prediction accuracy was calculated as the correlation between individual cows' phenotypes and their genomic predicted breeding values.

\section{Software and Parameter}

The 3 models described above (interaction, withinenvironment, and across-environment genomic BLUP models) were implemented using the "BGLR": Bayesian Generalized Linear Regression $\mathrm{R}$ package version 1.0.4 (Pérez and de los Campos, 2014). Gaussian priors, "BRR," with Bayesian models were assumed for marker effects. A total of 33,000 iterations were run, and the first 3,000 iterations were disregarded. Running additional iterations did not increase prediction accuracies. The remaining samples were thinned using default value (i.e., 5).

\section{RESULTS AND DISCUSSION}

\section{Assessment of Population Stratification}

Principal components were derived from the genomic relationship matrix of all cows using the $\mathrm{R}$ function eigen. The result was a matrix of eigenvectors with the number of rows equal to the number of animals and columns ordered by descending eigenvalues. These eigenvectors are referred to as principal components. The genetic substructure of the data was plotted using the 2 eigenvectors with the first and second largest eigenvalues, in Figure 1. The first 2 eigenvectors clustered animals into roughly 3 groups, and there were overlaps between groups. Animals from the same environment tended to cluster together, which may have led to in- 


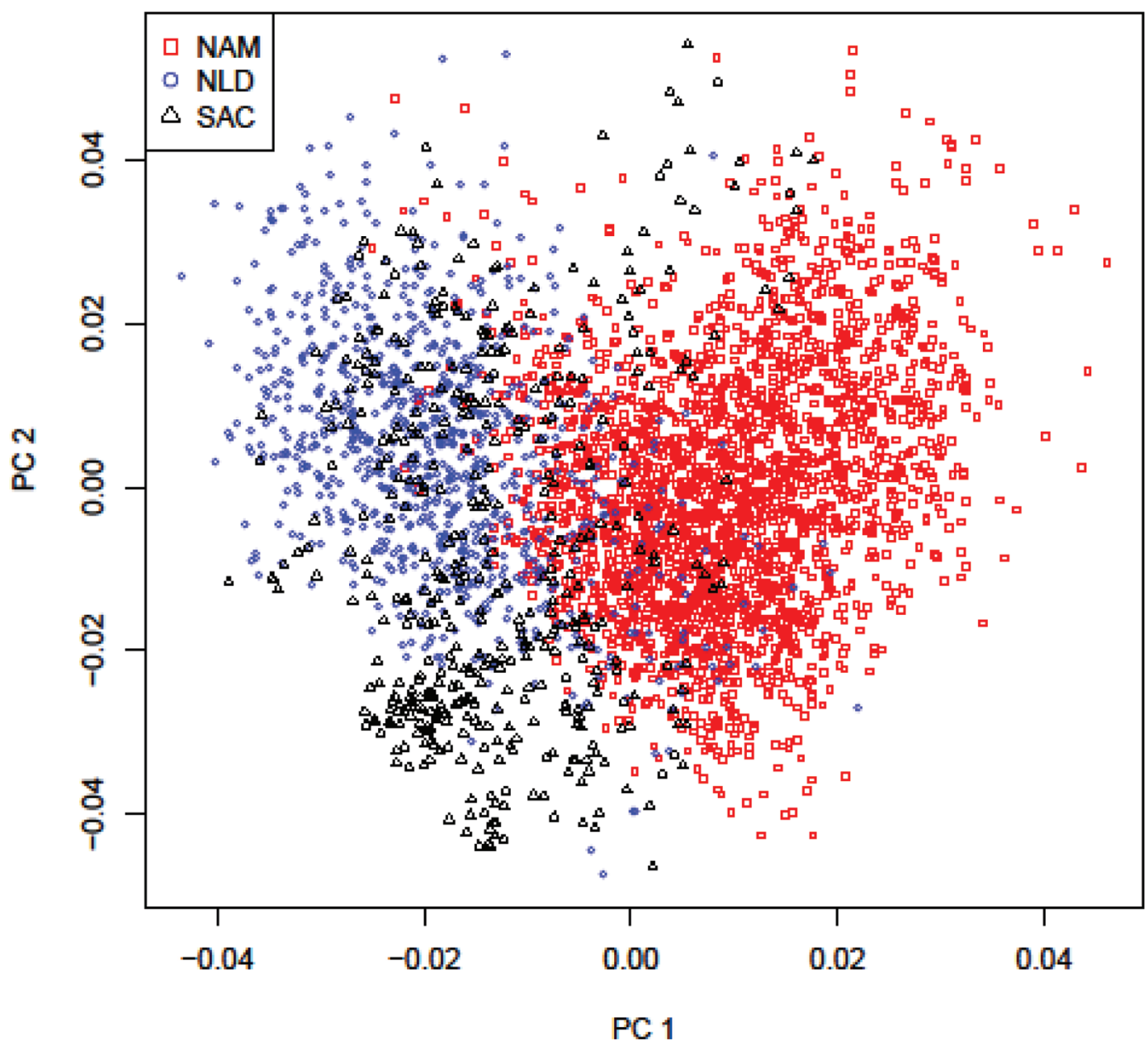

Figure 1. Plot of principal components (PC) 1 and 2 for all 3,656 genotyped animals, colored by their environments. NAM $=$ North America (square/red), NLD = the Netherlands (circle/blue), and SAC = Scotland (triangle/black). Color version available online.

teractions between genotypes and environment. Thus, genetic heterogeneity was present in the combined data set.

\section{Estimate of Genomic Variance Component}

Estimated genomic variance components for all traits and environments with each model are shown in Table 2. Within-environment model mostly gave smaller estimates of residual variances than the acrossenvironment analyses, which may indicate that the within-environment model had an improved fit to the data over the one that assumed constant marker effects across environments. The interaction model provided estimates of residual variance similar to those of the within-environment analyses. For instance, the estimate of residual variance for RFI in NLD was lowest for the within-environment model, intermediate for the inter- action model, and highest for the across-environment model.

As noted by de los Campos et al. (2015), an appealing feature of the interaction model is that it decomposes the genomic variance into the main and interaction variances, and the average proportion of genomic variance explained by main effects can be computed. The genomic variance parameter in the across-group model, however, was the same for all environments, whereas separate genomic variances were fitted for each environment in the within-environment model. The proportion of variance explained by the main effect can be computed from the ratio of the variance of the main effect to the sum of the variance of the main effects and the interaction variance, and ranged from 30 to $50 \%$ in the present study according to Table 2 .

Estimated genomic heritabilities for all traits were moderate to high. In general, estimated heritabilities 
YAO ET AL.

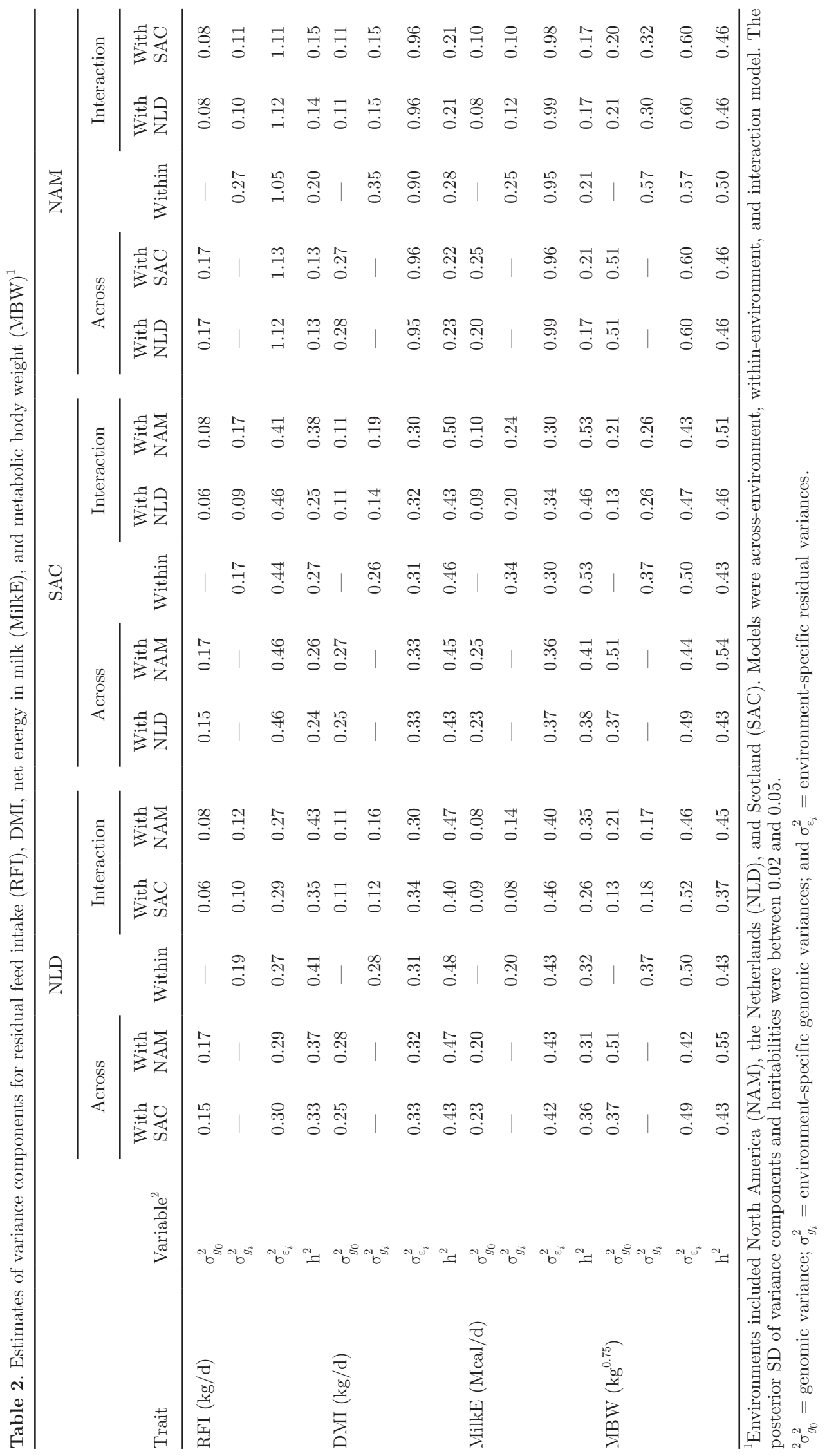


were the lowest for RFI (mean $=0.15$ ), ranging from 0.13 to 0.41 , and were the highest for MBW (mean $=0.46)$, ranging from 0.37 to 0.55 . The estimates for DMI $($ mean $=0.38)$ and MilkE $($ mean $=0.32)$ were similar, ranging from 0.21 to 0.50 and 0.17 to 0.53 , respectively. Though the definition of RFI varied in different studies, the estimated heritabilities of RFI in our study fell within the range of 0.00 to 0.32 for lactating dairy cattle as reported by Pryce et al. (2014). The estimates of MBW and DMI were also consistent with the ranges of 0.38 to 0.45 and 0.21 to 0.37 , respectively, as reported in Koenen and Veerkamp (1998) for a similar stage of lactation.

For all traits, the genomic variances were similar among 3 environments, but the differences in estimated residual variances across environments were large. The genomic variances were either similar or slightly larger in NAM than in NLD and SAC. For example, for RFI, the estimated genomic variances in NAM ranged from 0.17 to 0.27 , whereas the estimates in NLD and SAC ranged from 0.15 to 0.20 and 0.15 to 0.25 , respectively. The residual variances for RFI, DMI, and MilkE were 2 to 3 times larger in NAM than in NLD and SAC, with NAM (NLD and SAC) showing the worst (best) model fit for all models. Consequently, the estimated heritabilities were lower in NAM than other environments for RFI, DMI, and MilkE. Manzanilla-Pech et al. (2016) also reported that the estimated heritabilities for feed intake-related traits (RFI and DMI) in the United States were lower than those in NLD, but their estimates tended to be larger than in the present study. Country-specific estimated heritabilities for RFI were also reported by Tempelman et al. (2015) based on random regression models using only phenotypic data. The range of estimated heritabilities for NAM in the present study was comparable to that of (Tempelman et al., 2015), but the ranges for NLD and SAC in that study were smaller than those of the present study.

Genomic correlations between environments were also computed using the variance component estimates from the interaction model (Table 3), ranging from 0.36 between NAM and SAC for RFI to 0.47 between NAM and NLD for MBW. Averaged across all traits, genomic correlation was highest (0.42) between NAM and NLD and lowest (0.39) between NAM and SAC. Averaged across all environments, genomic correlations for DMI (0.43) and MBW (0.42) were higher than those for RFI (0.39) and MilkE (0.39). In other words, cows from NAM and NLD shared more genomic similarities. However, these 2 populations were not shown to be closely related. The reason could be that the similarity may not be strong enough to be shown with the relatively large standard deviations of the estimated correlations
Table 3. Genomic correlations (mean \pm SD) between different pairs of environments for residual feed intake (RFI), DMI, net energy in milk (MilkE), and metabolic body weight (MBW); environments were North America (NAM), the Netherlands (NLD), and Scotland (SAC)

\begin{tabular}{lccc}
\hline Item & NLD-SAC & NLD-NAM & SAC-NAM \\
\hline RFI (kg/d) & $0.39 \pm 0.11$ & $0.41 \pm 0.08$ & $0.36 \pm 0.08$ \\
DMI (kg/d) & $0.46 \pm 0.13$ & $0.43 \pm 0.10$ & $0.39 \pm 0.10$ \\
MilkE (Mcal/d) & $0.40 \pm 0.10$ & $0.38 \pm 0.08$ & $0.39 \pm 0.08$ \\
MBW (kg $\left.\mathrm{kg}^{0.75}\right)$ & $0.38 \pm 0.12$ & $0.47 \pm 0.13$ & $0.40 \pm 0.12$ \\
\hline
\end{tabular}

(about 0.1). Notably, the data from SAC and NLD were collected on older animals (born between 1983 and 2008) relative to NAM (born between 1999 and 2010), and the genomic correlations may reflect the genetic difference across generations. Compared with results of de Haas et al. (2015), the estimates of correlations for DMI in our study were generally smaller, but the lowest correlations were similarly found between SAC and NAM (the cows tested at Iowa State University research station).

\section{Accuracy of Genomic Prediction}

Table 4 shows the average environment-specific correlations between predictions and observations from 10 training-testing partitions for each trait and model. Average correlations achieved across models increased with estimated heritabilities of the traits. The traits with the lowest to the highest accuracies were RFI (mean accuracy $=0.19$; mean heritability $=0.15)$, Mil$\mathrm{kE}($ mean accuracy $=0.33$; mean heritability $=0.32$ ), DMI (mean accuracy $=0.36$; mean heritability $=0.38$ ), and MBW (mean accuracy $=0.38$; mean heritability $=0.46)$. This trend was consistent with Goddard and Hayes (2009), who stated that, given the same reference population size, the traits with higher estimated heritabilities tended to have more accurate model predictions. Those authors also concluded that a larger reference population might be needed for traits with lower heritabilities, such as RFI, to achieve comparable prediction accuracies.

Among the 3 models, the correlations varied among traits, and no single model was the best across traits and environments. The interaction model gave the highest prediction accuracy for MBW, which had the highest estimated heritabilities ranging from 0.37 to 0.55 . The within-environment model performed the best when predicting RFI, which had the lowest estimated heritabilities, ranging from 0.13 to 0.41 . For traits (DMI and MilkE) that had intermediate estimated heritabilities ( 0.21 to 0.50 and 0.17 to 0.53 , respectively), the performance of 3 models was comparable. In other 
YAO ET AL.

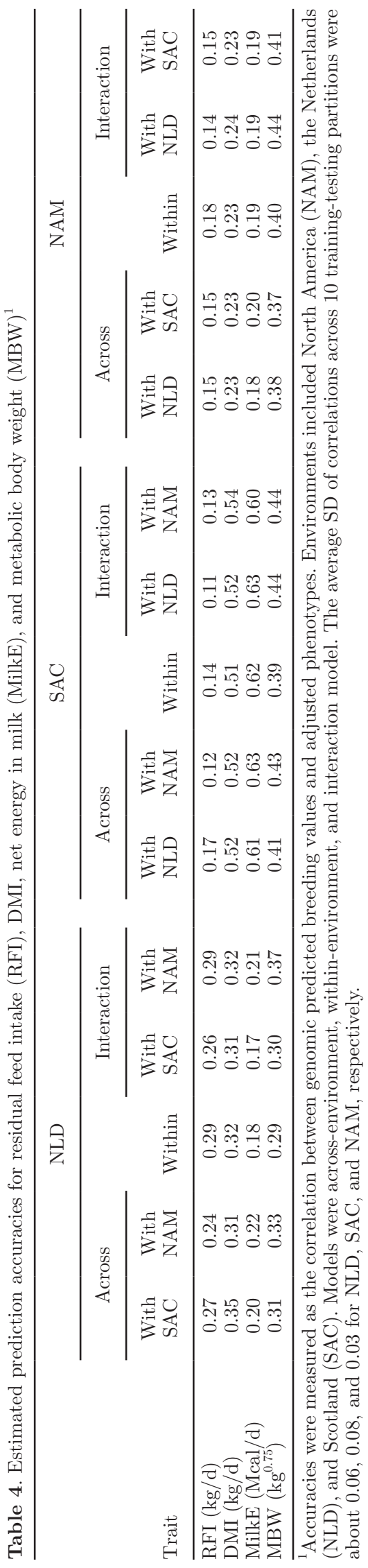

words, combining data from different environments did not contribute to the improvement in prediction accuracies for RFI using either the interaction model or across-environment model, though it led to increases in accuracies for DMI (up to 3\%), MilkE (up to 4\%), and MBW (up to 8\%) compared with the within-environment analyses. The increase in accuracy for DMI was also demonstrated by de Haas et al. (2012) using a multitrait model when pooling data from Australia, the United Kingdom, and the Netherlands. Overall, the interaction model either was the best-performing method or, more often, tended to perform nearly as well as the best method, which was consistent with results from de los Campos et al. (2015).

Lastly, substantial differences were observed among prediction accuracies in different environments, when averaged across models. Among the 3 environments, the one that had the largest estimated heritabilities achieved the best prediction accuracies. Surprisingly, the influence of the reference population size was not reflected in prediction accuracies. With the largest reference population, accuracies in NAM were not the best for any trait; SAC, with the smallest reference population, had the highest accuracies in all traits, except RFI due to the largest estimated heritabilities of traits. The reason for this could be that data from NAM were combined from multiple experiments and cows were fed different diets in each experiment. Additionally, cows in NAM were from 7 research stations located in different parts of the United States and Canada, which may have different breeding and management strategies. Data from SAC were derived from 2 research stations. Herds in SAC and NLD were each involved in only 2 different selection lines. Therefore, both genetic and environmental variations in data from NAM could be greater than that of the other 2 environments.

Overall, the 3 clusters in Figure 1 shows evidence of heterogeneity of genotypes, and the differences between estimates of variance components from each model indicated the heterogeneity of the marker effects. The across-environment analyses provided estimates of residual variance that were mostly higher than those obtained with the within-environment analyses. This difference suggested that the ability of the model to fit the data was hampered by forcing marker effects to be constant across environments. The interaction model gave estimates of residual variances that were similar to those of the within-environment analyses. In prediction accuracy, differences due to the model used were relatively small. When averaged across traits and environment, however, we found a slight superiority of the interaction model over the across-environment and within-environment analyses, which was consistent with results from de los Campos et al. (2015). This might 
have been cause by the interaction model being more robust than the other 2 models due to its flexibility of handling the proportion of genomic variance explained by the main effect, no matter whether the estimated correlations of effects between environments were high or low, as discussed by de los Campos et al. (2015).

\section{CONCLUSIONS}

With a specific application to RFI and its component traits, we constructed an interaction whole-genome regression model that provided a novel way to evaluate traits measured in multiple environments where genetic heterogeneity may exist. Compared with the across-environment and within-environment models, we showed that the interaction model was capable of estimating environment-specific genomic parameters. Overall, the interaction model performed better or nearly equal to the best alternative in terms of accuracy of the genomic prediction. Combining data from 3 environments (NAM, NLD, and SAC) provided increases in prediction accuracies for DMI, MilkE, and MBW, but not much for RFI, when using either the interaction model or the across-environment model. The interaction model may be more robust for application to novel traits for which the cost or labor associated with data collection necessitates pooling reference populations across multiple environments (or countries). However, simplifying assumptions or constraints may be necessary to avoid over parameterization if multiple subpopulations exist.

\section{ACKNOWLEDGMENTS}

This project was supported by Agriculture and Food Research Initiative Competitive Grants no. 2008-3520518711 and 2011-68004-30340 from the USDA National Institute of Food and Agriculture (Washington, DC). Support from Hatch grant no. MSN139239 from the Wisconsin Agricultural Experiment Station (Madison, WI) is acknowledged, and K. A. Weigel acknowledges partial financial support from the National Association of Animal Breeders (Columbia, MO).

\section{REFERENCES}

Bates, D., M. Maechler, B. Bolker, and S. Walker. 2013. Lme4: Linear mixed-effects models using eigen and s4. $\mathrm{R}$ package version $1(4)$. Accessed Jan. 11, 2017. https://cran.r-project.org/web/packages/ lme4/index.html.

Berry, D. P., M. Coffey, J. Pryce, Y. De Haas, P. Løvendahl, N. Krattenmacher, J. Crowley, Z. Wang, D. Spurlock, and K. Weigel. 2014. International genetic evaluations for feed intake in dairy cattle through the collation of data from multiple sources. J. Dairy Sci. 97:3894-3905.

Crossa, J., G. de los Campos, M. Maccaferri, R. Tuberosa, J. Burgueño, and P. Pérez-Rodríguez. 2015. Extending the marker $\times$ environment interaction model for genomic-enabled prediction and genome-wide association analysis in durum wheat. Crop Sci. 56:2193-2209. https://doi.org/10.2135/cropsci2015.04.0260.

Davis, S. R., K. Macdonald, G. Waghorn, and R. Spelman. 2014. Residual feed intake of lactating holstein-friesian cows predicted from high-density genotypes and phenotyping of growing heifers. J. Dairy Sci. 97:1436-1445.

de Haas, Y., M. Calus, R. Veerkamp, E. Wall, M. Coffey, H. Daetwyler, B. Hayes, and J. Pryce. 2012. Improved accuracy of genomic prediction for dry matter intake of dairy cattle from combined European and Australian data sets. J. Dairy Sci. 95:6103-6112.

de Haas, Y., J. Pryce, M. Calus, E. Wall, D. Berry, P. Løvendahl, N. Krattenmacher, F. Miglior, K. Weigel, and D. Spurlock. 2015. Genomic prediction of dry matter intake in dairy cattle from an international data set consisting of research herds in Europe, North America, and Australasia. J. Dairy Sci. 98:6522-6534.

de los Campos, G., Y. Veturi, A. I. Vazquez, C. Lehermeier, and P. Pérez-Rodríguez. 2015. Incorporating genetic heterogeneity in whole-genome regressions using interactions. J. Agric. Biol. Environ. Stat. 20:467-490.

Goddard, M. E., and B. J. Hayes. 2009. Mapping genes for complex traits in domestic animals and their use in breeding programmes. Nat. Rev. Genet. 10:381-391.

Hardie, L. C., L. Armentano, R. Shaver, M. VandeHaar, D. Spurlock, C. Yao, S. Bertics, F. Contreras-Govea, and K. Weigel. 2015. Considerations when combining data from multiple nutrition experiments to estimate genetic parameters for feed efficiency. J. Dairy Sci. 98:2727-2737.

Hayes, B. J., P. Bowman, A. Chamberlain, K. Verbyla, and M. Goddard. 2009. Accuracy of genomic breeding values in multi-breed dairy cattle populations. Genet. Sel. Evol. 41:51.

Karoui, S., M. J. Carabaño, C. Díaz, and A. Legarra. 2012. Joint genomic evaluation of French dairy cattle breeds using multiple-trait models. Genet. Sel. Evol. 44:39.

Koenen, E. P. C., and R. F. Veerkamp. 1998. Genetic covariance functions for live weight, condition score, and dry-matter intake measured at different lactation stages of Holstein Friesian heifers. Livest. Prod. Sci. 57:67-77. https://doi.org/10.1016/S03016226(98)00159-6.

Lehermeier, C., C.-C. Schön, and G. de los Campos. 2015. Assessment of genetic heterogeneity in structured plant populations using multivariate whole-genome regression models. Genetics 201:323-337.

Lopez-Cruz, M., J. Crossa, D. Bonnett, S. Dreisigacker, J. Poland, J.-L. Jannink, R. P. Singh, E. Autrique, and G. de los Campos. 2015. Increased prediction accuracy in wheat breeding trials using a marker $\times$ environment interaction genomic selection model. G3 (Bethesda) 5:569-582. https://doi.org/g3.114.016097.

Manzanilla-Pech, C. I., R. Veerkamp, R. Tempelman, M. van Pelt, K. Weigel, M. VandeHaar, T. Lawlor, D. Spurlock, L. Armentano, and C. Staples. 2016. Genetic parameters between feed-intakerelated traits and conformation in 2 separate dairy populationsThe Netherlands and United States. J. Dairy Sci. 99:443-457.

NRC. 2001. Nutrient Requirements of Dairy Cattle. 7th ed. Natl. Acad. Sci., Washington, DC.

Olson, K. M., P. VanRaden, and M. Tooker. 2012. Multibreed genomic evaluations using purebred Holsteins, Jerseys, and Brown Swiss. J. Dairy Sci. 95:5378-5383.

Pérez, P., and G. de los Campos. 2014. Genome-wide regression and prediction with the bglr statistical package. Genetics 198:483-495. https://doi.org/10.1534/genetics.114.164442.

Pryce, J. E., J. Arias, P. Bowman, S. Davis, K. Macdonald, G. Waghorn, W. Wales, Y. Williams, R. Spelman, and B. Hayes. 2012. Accuracy of genomic predictions of residual feed intake and 250day body weight in growing heifers using 625,000 single nucleotide polymorphism markers. J. Dairy Sci. 95:2108-2119.

Pryce, J., W. Wales, Y. De Haas, R. Veerkamp, and B. Hayes. 2014. Genomic selection for feed efficiency in dairy cattle. Animal 8:110. https://doi.org/10.1017/S1751731113001687.

Tempelman, R. J., D. Spurlock, M. Coffey, R. Veerkamp, L. Armentano, K. Weigel, Y. de Haas, C. Staples, E. Connor, and Y. Lu. 2015. Heterogeneity in genetic and nongenetic variation and energy sink 
relationships for residual feed intake across research stations and countries. J. Dairy Sci. 98:2013-2026.

Van Arendonk, J., G. Nieuwhof, H. Vos, and S. Korver. 1991. Genetic aspects of feed intake and efficiency in lactating dairy heifers. Livest. Prod. Sci. 29:263-275.

Veerkamp, R., G. Emmans, A. Cromie, and G. Simm. 1995. Variance components for residual feed intake in dairy cows. Livest. Prod. Sci. 41:111-120.

Wiggans, G., T. Cooper, D. Null, and P. VanRaden. 2014. Increasing the number of single nucleotide polymorphisms used in genomic evaluations of dairy cattle. Proc. 10th World Congr. Genet. Appl. Livest. Prod., Vancouver, Canada, Comm 301.

Williams, Y. J., J. Pryce, C. Grainger, W. Wales, N. Linden, M. Porker, and B. Hayes. 2011. Variation in residual feed intake in
Holstein-Friesian dairy heifers in southern Australia. J. Dairy Sci. 94:4715-4725.

Yao, C., L. Armentano, M. VandeHaar, and K. Weigel. 2015. Short communication: Use of single nucleotide polymorphism genotypes and health history to predict future phenotypes for milk production, dry matter intake, body weight, and residual feed intake in dairy cattle. J. Dairy Sci. 98:2027-2032.

Yao, C., D. Spurlock, L. Armentano, C. Page, M. VandeHaar, D. Bickhart, and K. Weigel. 2013. Random forests approach for identifying additive and epistatic single nucleotide polymorphisms associated with residual feed intake in dairy cattle. J. Dairy Sci. 96:6716-6729. 